\title{
Journal changes and initiatives
}

\author{
Ryan E. Walther, MA, and Richard D. Weisel, MD
}

\author{
From The American Association for Thoracic Surgery, Beverly, Mass; and the Division of Cardiovascular Sur- \\ gery, Toronto General Hospital, Research Institute and the University of Toronto, Toronto, Ontario, Canada. \\ Disclosures: Authors have nothing to disclose with regard to commercial support. \\ Address for reprints: Richard D. Weisel, MD, Division of Cardiovascular Surgery, Toronto General Hospital, \\ Research Institute and the University of Toronto, 200 Elizabeth St 4N-476, Toronto, Ontario, Canada M5G \\ 2C4 (E-mail: rweisel@uhnresearch.ca). \\ J Thorac Cardiovasc Surg 2016;151:328-9 \\ $0022-5223 / \$ 36.00$ \\ Copyright (C) 2016 by The American Association for Thoracic Surgery \\ http://dx.doi.org/10.1016/j.jtcvs.2015.12.026
}

The Journal is continually seeking to improve not only the quality of the information we publish but also how this information is presented and made available to readers. Therefore, beginning with this issue, we are launching several initiatives in 2016. The February issue is the debut of a new front page for original articles featuring a $\mathbf{Q R}$ code. QR is short for Quick Response because they can be read quickly by a smartphone and are seen often in advertisements. Once scanned by a smartphone or tablet, the code will take your browser to a URL displaying the video(s) associated with the article or other supplemental data (eg, appendices, data sets, or supplemental figures and tables), or the article title page if there are no additional data to access. Readers need to download a $\mathrm{QR}$ reader app for their smartphone to use this functionality, either at iTunes for an iPhone (Apple, Cupertino, Calif) or the Google Play Store (Google Inc, Mountain View, Calif) for Android phones. As 2016 progresses, all original articles will be displayed in this format. We have added this feature for 2 primary reasons: To make our online information more accessible and user-friendly and to encourage the submission of more visually appealing online content for each article.

To that end, our intention is that all articles will be accompanied by a video, a process that we will initiate in 2016 and hopefully achieve in 2017. Authors will be asked to submit a video highlighting the topic of the manuscript for all original articles. In 2015, we began adding video links to the meeting presentations of all articles originating from the American Association for Thoracic Surgery Annual Meeting (a program we plan to expand to other American Association for Thoracic Surgery meetings, eg, the Aortic Symposium and Mitral Conclave). Videos could be procedural, informational, interviews, or other relevant material. The recommended size limit for videos is $150 \mathrm{MB}$ (although we can accommodate larger file sizes on request). Depending on file format and compression, this size corresponds to 15 minutes for regular-definition videos or 5 minutes for high-resolution, high-definition format. A guide for creating acceptable videos for the

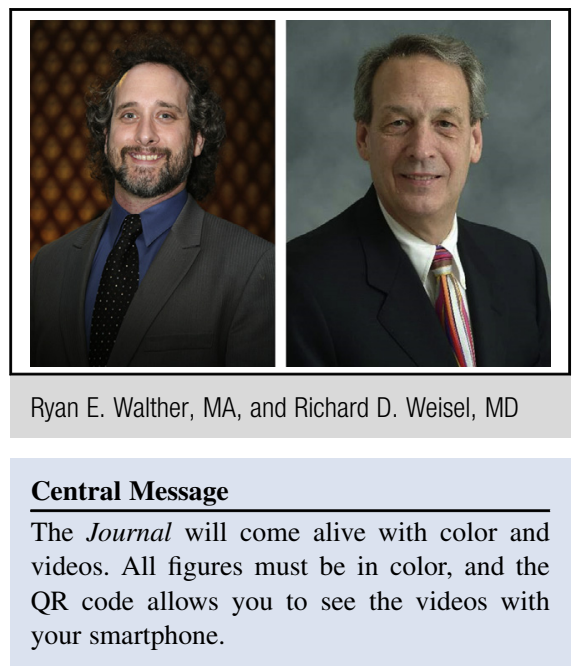

Journal will soon appear on our manuscript submission Web site.

It is not just in the realm of video that we are making submission changes. The Journal now requires color for all figures. Journal staff are creating templates to assist authors, as well as a color palette that authors can use. This new process will enable the Journal to create a consistent style, look, and feel for our illustrations.

We also have adjusted the Journal section names. You will notice in this issue new tab abbreviations and table of contents formats have been instituted. The 3 primary journal sections have been shortened to Acquired, Congenital, and Thoracic to replace the previous longer and unwieldy titles. The sections will be easier to reference and offer clearer guidance to the content readers want to read. These same changes have been reflected in our online submission system and on the Journal Web site.

To offer further guidance, the Journal also will be inserting logos into the Journal that represent subspecialties. The Journal launched in November a contest to create logos, and we are finalizing the designs. These logos will represent the various sections of the Journal and will be used both online and in print as visual references, indicating the topics contained in an article and where topics overlap (eg, an article on cardiac transplantation would contain the logo for both Transplantation and Acquired).

One last change of note concerns the format of the Journal online. The Journal has collaborated with Elsevier this past year to create a new, automated, and very robust taxonomy for the content in the Journal, and also pulled in content from Seminars in Thoracic and Cardiovascular 
Surgery, Operative Techniques in Thoracic and Cardiovascular Surgery, and The Pediatric Cardiac Surgery Annual. The new taxonomy was necessary to launch our new collections. The previous online collections were outdated and neither intuitive nor user-friendly. The new collections, which appear as a second navigation bar at jtcvsonline.org, provide a unique experience for each of our subspecialties. Each collection provides the latest information published from all 4 journals on nearly every area of interest, such as aorta, mitral valve, mechanical circulatory support, hypoplastic left heart syndrome, lung cancer, lung transplantation, and so forth. The new collections can be individualized by the reader, but the default is to provide the most recent material first. The collections are easier to navigate, better reflect current practice, and will be auto-populated on the basis of the new taxonomy. Each of the major subspecialties has multiple layers, so users can identify their topics of interest more easily. Top-level collections are Acquired, Congenital, Thoracic, Basic Science/Innovation, Education, and Perioperative.

We hope these initiatives provide more information for readers, increase the ease of discoverability and usability of our online and print formats, and, not to be discounted, are more aesthetically pleasing. Continual progress is our goal, but, as always, we cannot progress in isolation. Readers always should feel free to contact the editorial office with ideas for improving the Journal. We look forward to your ideas and feedback as the year progresses. 